\title{
WSN and RFID Integration to Support Intelligent Monitoring in Smart Buildings Using Hybrid Intelligent Decision Support Systems
}

\author{
F. Alshahrany ${ }^{a, *}$, M. ABbod ${ }^{a}$ And I. MounleK ${ }^{b}$ \\ ${ }^{a}$ College I of Engineering Design and Physical Sciences, Brunel University London, \\ Uxbridge UB8 3PH, United Kingdom \\ ${ }^{b}$ Hybrid Intelligent Decision Systems, Jerjer House, Nottingham, United Kingdom
}

\begin{abstract}
The real time monitoring of environment context aware activities is becoming a standard in the service delivery in a wide range of domains (child and elderly care and supervision, logistics, circulation, and other). The safety of people, goods and premises depends on the prompt reaction to potential hazards identified at an early stage to engage appropriate control actions. This requires capturing real time data to process locally at the device level or communicate to backend systems for real time decision making. This research examines the wireless sensor network and radio frequency identification technology integration in smart homes to support advanced safety systems deployed upstream to safety and emergency response. These systems are based on the use of hybrid intelligent decision support systems configured in a multi-distributed architecture enabled by the wireless communication of detection and tracking data to support intelligent real-time monitoring in smart buildings. This paper introduces first the concept of wireless sensor network and radio frequency identification technology integration showing the various options for the task distribution between radio frequency identification and hybrid intelligent decision support systems. This integration is then illustrated in a multi-distributed system architecture to identify motion and control access in a smart building using a room capacity model for occupancy and evacuation, access rights and a navigation map automatically generated by the system. The solution shown in the case study is based on a virtual layout of the smart building which is implemented using the capabilities of the building information model and hybrid intelligent decision support system.
\end{abstract}

DOI: 10.12693/APhysPolA.128.B-152

PACS: 07.05.Tp

\section{Introduction}

The fast development of communications and ubiquitous computing results in integrating communication and computing capabilities in common intelligent objects of everyday use, creates a new generation of pervasive networks. Sensors are one of these objects, and their use in surveillance and monitoring activities has led to the development of hybrid intelligent networks based on sensor integration in tags and objects, taking advantage of their radio frequency identification (RFID) identifying tracking capabilities to capture real time data needed for instantaneous decisions.

In RFID technology, tags are used to store data and readers to retrieve this data from the tags, whereas wireless sensor network (WSN) technology involves small smart interconnected sensing devices with extensive wireless communication facilities. From their early stages, RFID systems have been successfully used for animal tagging [1] and road toll collection [2] and WSN systems in environmental monitoring [3] and robotics [4], and WSNs based on mote sensing platforms have been used for the more varied applications.

\footnotetext{
* corresponding author; e-mail: Falah. Alshahrany@brunel.ac.uk
}

Nowadays, integrated RFID and WSN are used in combination of each other, incorporating intelligent fixed and mobile devices in asset tracking and security, resulting in novel applications covering a variety of industrial and commercial domains. These include people and objects identities and locations, collect objects environmental conditions and mobile and networking services distribution. The integration of these two key technologies which are an essential component support of ubiquitous and pervasive computing $[5,6]$, aims at merging their individual abilities to store and retrieve data through electromagnetic transmission using an RF compatible circuit, collect, aggregate and analyze environmental real time data about objects, their localisation and environmental conditions. The merge of these individual capabilities is a conceptual specification to meet the primary requirement of compensating the lack of intelligence within the behavioural monitoring process and the environmental conditions of the organisational setting to control its real time events. The benefits of integration are overwhelming.

This paper presents a glimpse of how this integration can be supported by an intelligent decision support system to support access management, identifying and tracking. It starts by a brief summary of related work and presents the research motivation. Section 4 describes the conceptual framework by listing the key issues discussed in this work, the WSN-WSN integration 
specifications, presenting the WSN-RFID integrated framework, and presents the different fundamental integration levels: physical and functional and logical (software). Finally, Sect. 5 presents a case study of WSNRFID integration implemented as the sensing and tracking component of the hybrid intelligent decision support systems (HIDSS).

\section{Related work}

Integration classes and platforms have been extensively discussed in literature [7-11], and integration issues are inherent to the lack of consensus among professionals about the integration approach. The provision of context information between people and objects is a key requirement for the design and delivery of real time context aware services provided in so-called smart spaces for the intelligent monitoring of people and smart objects behaviour circumstances and conditions. The integration of the WSN and RFID technologies provides sensor data to the RFID infrastructure, taking advantage of the tags unique identifiers to enhance manageability and add value to the web-based RFID core services [12], creating smart objects.

These services require supporting interoperability for the main realtime data services which include collecting, storing, manipulating and analysing, and retrieving data from integrated homogeneous and heterogeneous environments. The development of support functions integrated in the WSN-RFID middleware has been suggested in several context aware applications [13-16]. Integrating sensors in enhanced sensors tags has been proposed in different wireless identification sensing platform (WISP) configurations or implemented in various context applications $[7,17]$.

\section{Motivation}

The focus in this work is the integration of the support interoperability for the main realtime data services in the design of a HIDSS to meet the requirements of the conceptual support model of the integrated RFID-WSN architecture. This support system which aims at hiding the complexity of accessing the sensor network environment and developing an application for integrated tag and sensor web enablement at an external user level, is to be implemented outside the middleware layer, with a clear separation of the service layer supporting the different domain applications from the service configuration of the integrated WSN and RFID system architecture.

The HIDSS module service configuration of WSNs in homogeneous and heterogeneous environment has been implemented [18, 19]. The integrated WSN and RFID context aware service configuration proposed in this work to enable configuration capabilities to support the four classes of integration, is a web-based architecture integrating composed smart services developed using intelligent agents orchestrating automatically the different WSN-RFID integrated entities. The orchestration process is to remain invisible to the system users.

\section{The conceptual framework}

\subsection{The key issues}

The research work presented in this paper addresses several key open issues listed below:

- The elaboration process for efficient real time data capture, integration and management, intelligent data transformation and aggregation, and intelligent capabilities for large-scale application support involving a high interaction between tagged fixed and mobile objects and people;

- The abilities limitation of RFID networks and services to fully encompass environment-sensitive objects and their varying environmental conditions, and capture and transmit real time sensing information, limiting the integration scope for sensors incorporation in tags and RFID readers in heterogeneous and other smart devices;

- The limited tag functionality and processing capabilities requiring more RFID readers in the reader coverage zone conveying the risk of tag readers collisions resulting in the tags incapability of decoding the readers commands;

- The fault tolerance performance to resist to nodes failure and RFID misreading and reading failures caused by RFID readers collisions which are known as interferences between reader to reader in the likelihood of readers collisions, and also between multiple reader to tags and

- The security threats for RFID dissociating the traditional and new threats.

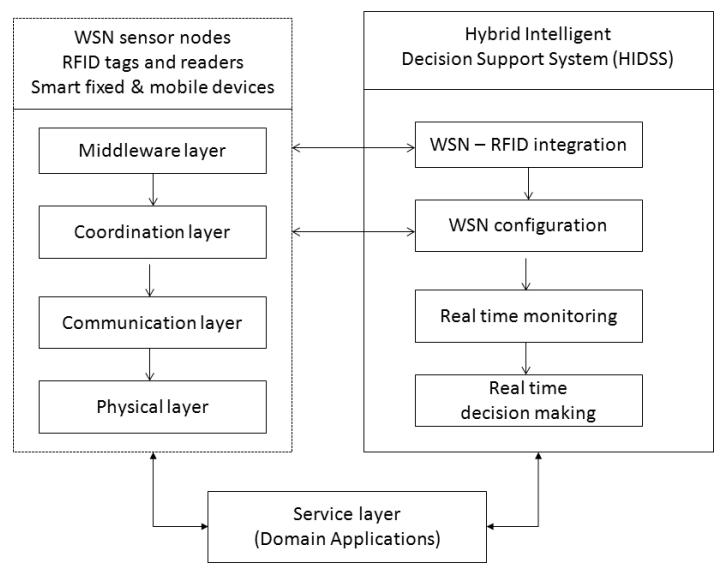

Fig. 1. System architecture.

These issues are discussed, and solutions to inherent problems are implemented in the support capabilities of the HIDSS at the four levels of the integrated RFIDWSN architecture model as illustrated in Fig. 1. However, other important issues related to the improvement of WISP in terms of tag power harvesting approaches, and tag sensors integration techniques are not included in the scope of this research work. 


\subsection{The WSN-RFID integration specifications}

The WSN-RFID integrated conceptual framework includes the following integration design specifications:

- The integration of RFID readers and devices to enhance the functionality of smart devices contributing to process integration and real time decision making;

- The integration of RFID tags and WSN sensor nodes to extend the tags applicability enhance the tags sensing capabilities and enable them to perform local tasks, turning them into intelligent and smart devices enabling intelligent monitoring;

- The replacement of WSN sensor nodes by using passive RFID tags for their low cost and very low energy power requirements, to enhance the sensors wireless capabilities particularly the tracking of the sensor nodes in non fixed WSN configuration;

- The extended capabilities of tags in terms of using the tag memory to store additional data, and the read and write to link the object individual data to the background database, and support the system data distribution required to enable the system local autonomy;

- The local deployment of the tag processing tasks versus providing tag long communication range due to the limited energy of sensors, compensated by effective wireless connectivity to exchange data between the tag sensor and the system.

\subsection{The WSN-RFID integrated framework}

The conceptual framework presented in this paper is about integrating mobile and fixed RFID, sensor nodes and WSNs effectively and transparently interconnected and exchanging context and control deployment information with homogeneous and heterogeneous devices. These devices are embedded and deployed in a predefined area in smart spaces in general, and smart closed places (homes, hospitals, workshop, and others) in particular, and elsewhere to support advanced intelligent safety systems based on context aware applications used in safety and emergency response. The configuration setting is supported by an HIDSS which is an hybrid intelligent decision support system composed of real time monitoring and decision making components as illustrated in Fig. 2.

These two technologies are based on the use of conventional sensors technologies. In WSNs, the sensors nodes energy capacity limitations can be compensated by the use of very cheap RFID tags for their nearby reading whereas their sensing capabilities are not as good as those of WSN sensor nodes. The WSN-RFID integration principle consists of equipping mobile objects and users with RFID tags and WSN to receive ubiquitous services according to their identity and real-time sensor/actuator

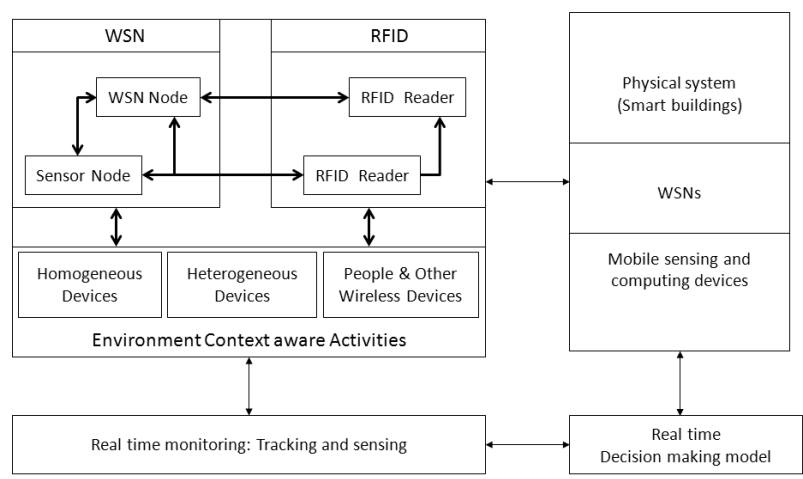

Fig. 2. WSN-RFID conceptual integration framework.

information. Integrating these two complementary technologies leads to bridge the detection and identification of objects with the real time intelligent monitoring of their environmental circumstances and conditions, and expands the overall functionality, capacity and performance of their devices.

\subsection{Physical integration}

The design specifications of sensor nodes and integrated WSN-RFID configurations depends on the requirements complexity for the use of different components of WSN, RFID and the context aware environment shown in Fig. 2. These requirements are derived from the application specifications for an intelligent real time monitoring of environment context aware activities which includes sensing of the environment, the tracking of objects, control of access and intrusion, and control of locking devices. This is supported by the integration of RFID tags and readers embedded in objects which results in the following four differents classes of WSN-RFID integration listed below.

- WSN sensors nodes and RFID readers;

- Homegeneous and heterogeneous devices, others wireless devices and people, and RFID readers;

- WSN sensors nodes, and RFID tags;

- Homegeneous and heterogeneous devices, other wireless devices and people, and RFID tags.

The cost and scale size reduction and the high level of integration and performance in such system configurations is the result of the tremendously rapid development of several technologies and techniques: computing, wireless communication, microprocessors, integrated circuits and RF identification. This development focuses more importantly on making these system configurations more open to integrate an additional range of varied smart devices and highly dynamic to react in real time to the environment conditions of change.

4.4.1. Unsensible devices sensing capabilities extension

Of great importance in the context aware computing applications is the ability extension of a sensor network to 
integrate trackability and sensible properties in unsensible objects; these properties include sensing and monitoring elements of context aware enviroments: sound, motion, pressure, temperature, humidity and others. In this work, we develop an application of both real time warning of proximity to hazardous objects and real time tracking of sensible zone acess. The integration of heat sensors in enhanced sensor tags using a wireless platform, and RFID readers into IP camera are the focus of the physical integration in this work, and the technical integration characteristics are developed in this section.

4.4.2. RFID tags, labels and readers embedded in objects

RFID Tags are microchips combined with a radio antenna, attached to objects or linked to people for identification and tracking. They give out periodically their unique electronic product code (EPC) via RF signal when they are in the read range of RFID readers. Readers enabled devices in smart buildings and elsewhere query and read EPC from tags via RF signal, update EPC in EPC information services which is part of the RFID configuration or a standalone system. In this research, EPC information services associate additional sensing, positioning, tracking data and other environmental changes data stored in a database installed in a backend system supporting various service platforms and applications.

Identifying people and objects via embedded or attached RFID tags is integrated with automatic identification and data capture as one method of RFID, procuring the advantage of long distance reading without being in the sight of the RFID reader. The integration of sensors as above mentioned, enable the provision of measured environmental data and information as a response to the signal sent by the RFID reader to the tag. More importantly, programmed data processor is included in the tag configuration instead of chip-wired logic for processing the transmission and sensor data.

\subsubsection{Active reader active tags (ARAT)}

Active readers are used in this work to generate interrogator signals when identification of people or objects occurs, i.e. located in interrogation zone or in readers covered zone.

Active readers which are wirelessly connected to the WSN, can enable complete standalone solutions, and procure enhanced distributed capabilities to deploy in both mobile and fixed site applications.

\subsubsection{Wireless identification sensing platform}

The sensing component of the conceptual framework is of the WISP that includes both sensing and computing. This platform is based on the use of a programmable passive or active reconfigurable RFID device (tags and other) capable of effectively integrating new dynamic computing tasks for configuration purposes in addition to easily accommodating the incorporation of a wide range of low power reconfigurable sensors (sensors enhanced RFID tags also called hybrid tags). The use of these tags is advocated for increased functionality for monitoring and objects or people tampering and complex conditions detection. The transmission of the sensor data is supported by the WSN wireless communication capabilities that integrate the RFID reader in the WSN configuration.

In this framework, identification and tracking is based on small RFID reader bracelets that report interaction with tagged objects to the HIDSS. The integration of WSN and RFID procures the flexibility of using passive tags to achieve high data and sensor activity rates, avoiding the active tags power supply limitations and inherent problems such as the batteries life time, their weight and volume, and cost. These hybrid passive tags are powered by RFID readers, and the sensing space is fully covered by these readers distributed or located using the hexagonal or square geographical pattern [20].

\subsection{Functional integration}

The tag mode in the use of passive versus active tags is of great significance in the determination of its specifications in terms of enabled reading capabilities (read only or read and write) and its functional integration specifications to link to databases using the ID number or use the tag programmable fields in the WISP to store the object-specific data or initiate a communication with other tags.

Active RFID tags with smart sensors are a high performance wireless transceiver device attached to people and objects in the smart building where the smart sensors detect the measured environmental and spatial conditions and changes. The tags transmit and receive data pertaining to their identification, access privileges of people or allowed movement zones of objects, and their location. Smart tags attached to people enable a hands free identification and access control with a continuous tracking of their location.

\subsubsection{Identification and tracking}

Active readers are integrated in IP cameras, and will receive ID information and timed location and frame of the person or object the tag is attached to. The received information is relayed to the server for the HIDSS to store and process.

The data received by the RFID reader is filtered before being sent to the server. The filtering process consists of the elimination of insignificant or irrelevant data and the elaboration of information should the RFID tag include a programmable processor. This process is performed using the middleware layer of the RFID software shown in Fig. 1.

Identification and tracking is supported by the integration of the RFID reader and IP camera. The service specifications include the deployment of a camera agent based on ID identification or other environmental event for:

- live video display or camera control,

- event triggered video recording and playback,

- automatic live video pop-up on alarm trigger, 
- search recorded video for playback,

- alarm activation and access control device parameters linked to CCTV cameras to start video cameras,

- display/alert doors and windows opening or blocking for use or not to use in emergency situations.

\subsubsection{Reading nearby tag over different distance ranges}

The RFID range is fundamental and the reading distance can be influenced by costs imposing for long range the use of active tags requiring a battery power supply.

Factors such as transmit power, receive sensitivity and efficiency, antenna, frequency, tag orientation and surroundings can considerably affect the specified range.

\subsubsection{Reading multiple tags}

Readers detect collision when reading multiple tags in their field of view: the present tags in the RFID reader coverage area broadcast their full IDs sharing the reader channel establishing a bi-directional communication using the singulation mechanism also called the tree walking. The reader establishes a tag reading sequence hierarchy using the ascending or descending order of the ID value. The encoded data of the selected tag is read and the tag set to sleep mode while reading the other colliding tags.

\subsection{Software integration}

The integration of the WSN and RFID technologies poses the problem of their modal interface when connecting them physically and deploying them in various system configurations. The system specifications in terms of services functionality depends on individual requirements of the several RFID, WSN, homogeneous and heterogeneous devices integrated in the system configuration.

\subsubsection{Integration requirements}

Of great importance in the integrated WSN-RFID conceptual framework are the RFID reader specifications that enable the creation of extended support capabilities to be configured in the HIDSS. The WSN mote is connected to the RFID reader to enable the mote to communicate with compatible devices. The data model is designed to effectively support the communication protocol when reading the different tags in use in the setting. This requires the development of elaborate multi-modal interfaces for a highly ubiquitous and pervasive computing to reduce the complexity of the implementation of such configurations and enhance the effectiveness and functionality of enabled services. The interface requirements are listed below:

- The integration of RFID tags and readers in the system configuration requires wireless or wired connection by USB/serial port between the reader, heterogeneous devices and the system to be opened using an application programming interface to implement a distributed unified interface;
- The data exchange between RFID tags and the system requires the storage in the system of all data of individual route tracing, displaying the individual detected position;

- Unique identifier per tag within multiple tag readings;

- The data filtering to eliminate insignificant data to be transmitted to the server;

- Full RFID reader zone coverage;

- Child detection in any part of the reader coverage zone;

- HIDSS must be able to generate and send alert to RFID tags (vibrating), smart computing devices and hand held devices via SMS and email;

- The RFID integrated system is deployed upon a WSN, and their deployment and configuration supported by HIDSS.

\subsubsection{WISP integration}

The interface integrates the WISP program that enables the communication between the platform and an RFID reader, and also the service measuring any attached sensors to the enhanced RFID tag. WISP is an open source that enables the implementation of the three following major functions:

- Sensor data communication between the WISP and a computer through an RFID reader using 64bit packets of sensor data including enhanced sensor tag power harvesting by the RFID reader, transmission from reader to WISP, and from WISP to reader.

- State and power management to activate or sleep a sensor, and

- Service protocol for encoding sensor data in the tag ID.

Of great importance in this platform is the number of measurements to be transmitted by tag ID for each reading, this number being associated to the number of sensors incorporated in the passive or active RFID tag. This number of measurement being limited, the sleep-active mode can be an appropriate sensor tag configuration factor to extend the number of sensors incorporated in the passive or active RFID tag enabled by the WISP programmability.

\subsubsection{Software components integration}

Three software components compose the software configuration as illustrated in Fig. 1. These components are integrated in the same configuration system supporting the delivery of end user services implemented in a distributed web or local network service architecture. 
- The integration of RFID and WSN technologies and their corresponding individual software (middleware, coordination and communication),

- HIDSS integrating the WSN-RFID interface and backend services, the WSN configuration, real time monitoring, and real time decision making, and

- Distributed domain applications and HIDSS.

\subsubsection{End-user services}

The end-user services are composed using a hierarchy of intelligent software agents and wirelessly connect smart devices, and configured to be invoked in different platforms.

\section{Case study}

The case study presented in this section will illustrate an intelligent emergency evacuation based on the use of an integrated WSN-RFID configuration in the context of surveillance activities in smart buildings. This component is an element of an emergency response system. A single building structure under a small WSN surveillance is modelled using the building information model to generate a virtual representation of the room's layout of a smart building equipped with RFID readers wirelessly connected to a small WSN $[18,19]$. In this modelling process, the details of the building layout and construction are used to derive concurrently the evacuation and surveillance devices requirements. The evacuation requirements are the basis for the study of the evacuation scenarios, whereas the surveillance devices requirements are needed to design the appropriate sensor nodes by selecting available sensors and their specifications. These nodes, once automatically localised, will be configured, clustered and linked to compose the WSN homogeneous nodes. The WSN similarly connects the heterogeneous devices which are also automatically allocated. The WSN homogeneous and heterogeneous sensor nodes are controlled for their deployment and configuration by intelligent configuration agents.

\subsection{Control access system}

HIDSS carries out an access control function to let in authorised people and let out those non identified or without room access privilege as illustrated in Fig. 3.

Privilege allocation in the control access system is a dynamic function that allocates for each person in the database dated and timed access to the building. For example, privilege "4" is represented by an hexadecimal value "0100" indicating for the ID 41767 an allowed access to rooms 1 and 2, and a denied access to room 3 .

HIDSS support the access filtering of ID tags in RFID readers as soon as an access privilege for a person has been allocated or changed. The date, time, location and privilege are stored in the database for each tag reading.

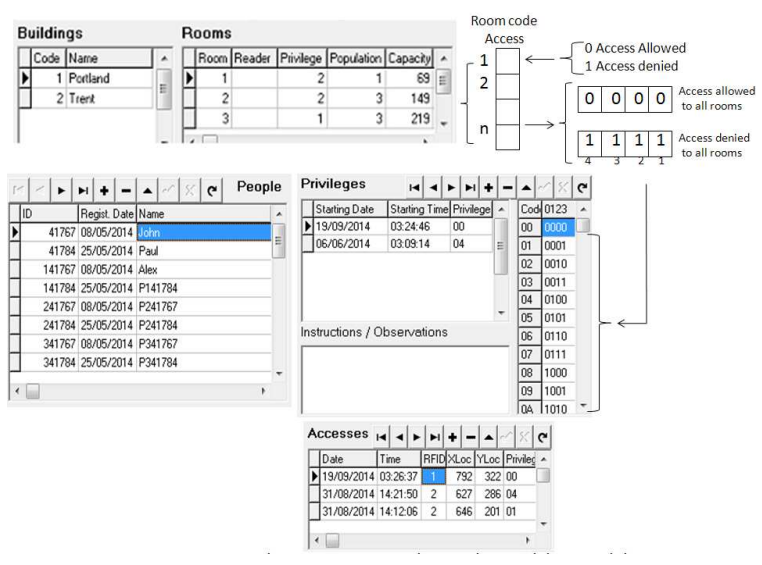

Fig. 3. People access room privilege coding and data model.

\subsection{Evacuation decision model}

People evacuation in the design of emergency response systems as a key element of emergency actions plans (EAP) and emergency procedures, is a prime requirement supported by a thorough risk assessment of the building safety. This assessment is validated by a decision model based on the use of room occupation norms and standards to set the rooms capacity in terms of accepted legal occupancy space requirements.
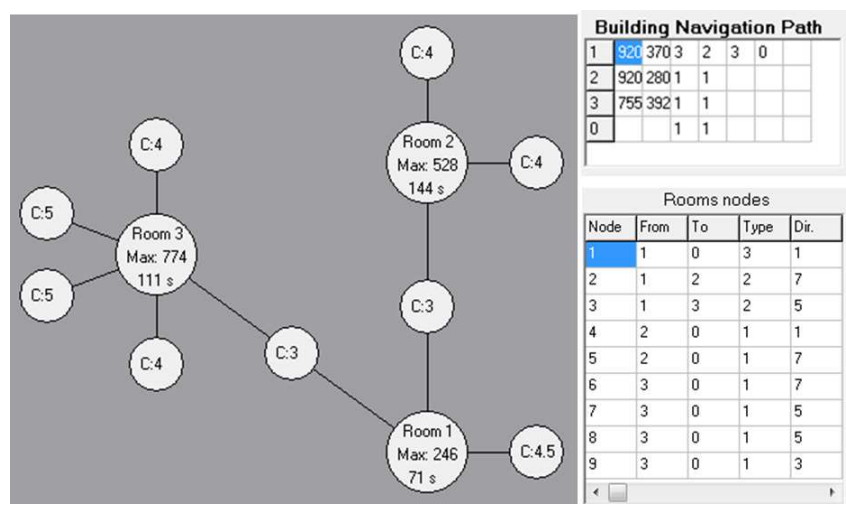

Fig. 4. Building navigation path and evacuation decision model.

The evacuation decision model shown in Fig. 4, requires the determination of the building navigation path and the identification and quantification of the rooms nodes corresponding to the potential evacuation exits to consider during the evaluation of plausible evacuation scenarios when preparing the evacuation plans aimed at responding to potential hazards, minimizing confusion and injuries and avoiding fatal outcomes. These elements are essential in elaborating the emergency escape routes and refining them in real-time as the hazards are occurring and developing. This requirement is supported by the HIDSS intelligent evacuation component which takes into account the speed of people leaving the rooms they are located in and the place of hazard occurrence and the 
hazard progression to refine individual paths to contour objects and hazards.

The identification of emergency escape routes is supported by basic requirements. An emergency escape route must:

- Be permanent, based on one or more exit accesses with unlocked doors and ground floor windows, and separated by file material resistant;

- Take into account the size of the building, its occupancy, the arrangement of the workplace and situational factors of both people, hazards and building;

- Satisfy the exit discharge, leading directly outside the building, and ensure that capacity must not decrease in the direction of exit route travel to the exit discharge, and

- Be associated to emergency safeguards including emergency equipments such as alarms, marked visible exit signs, and sprinklers to be deployed during the emergency response.

A varied type of heterogeneous devices are used along the hazards detectors called homogeneous devices to compose the smart building emergency equipments shown in Fig. 5.

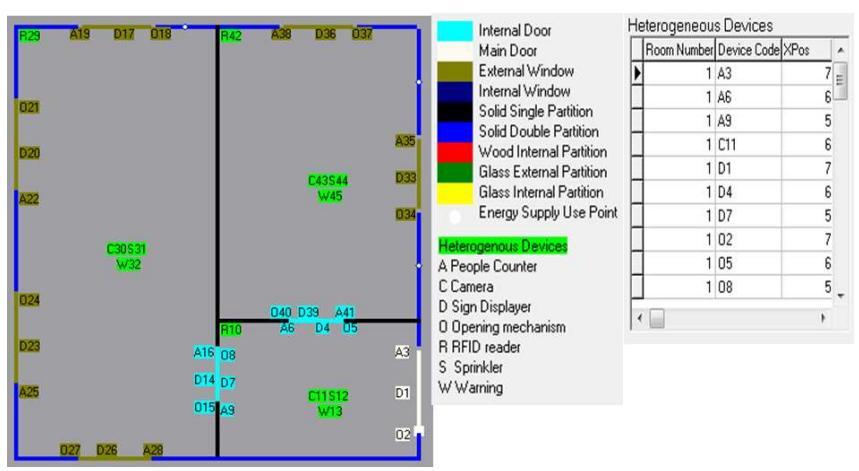

Fig. 5. Heterogeneous devices in use during emergency response.

In this work, the design of emergency escape routes is supported by the use of a knowledge base producing elaboration rules stored in a model base. The rule implemented in this case study to design an escape route is based on three concepts: exit, exit access and exit discharge. An exit access is a portion of an emergency escape route that leads to an intermediary exit or a final destination considered to be the exit discharge, an open space outside the building.

In this implementation, internal doors represent exits, the external door is the exit discharge, and the exit accesses are line segments corresponding to route portions from the initial localisation of people to internal doors and from internal doors to external doors.

\subsection{Emergency escape routes}

The identification of people and their individual localisation is used in HIDSS to determine emergency escape routes as illustrated in Fig. 6 showing objects or hazards avoidance using the tag sensor data transmitted to the back-end system by the rooms RFID readers. Collision detection is used to refine the emergency escape route to ensure to everyone a continuous and unobstructed path of exit travel from the point of their localization inside the building to a place of safety (exit discharge), as indicated in emergency action plans. Route guidance is individually provided to the rooms' attendants using voice messages to support the route guidance facilitation in adverse conditions people disability and when visibility is reduced.
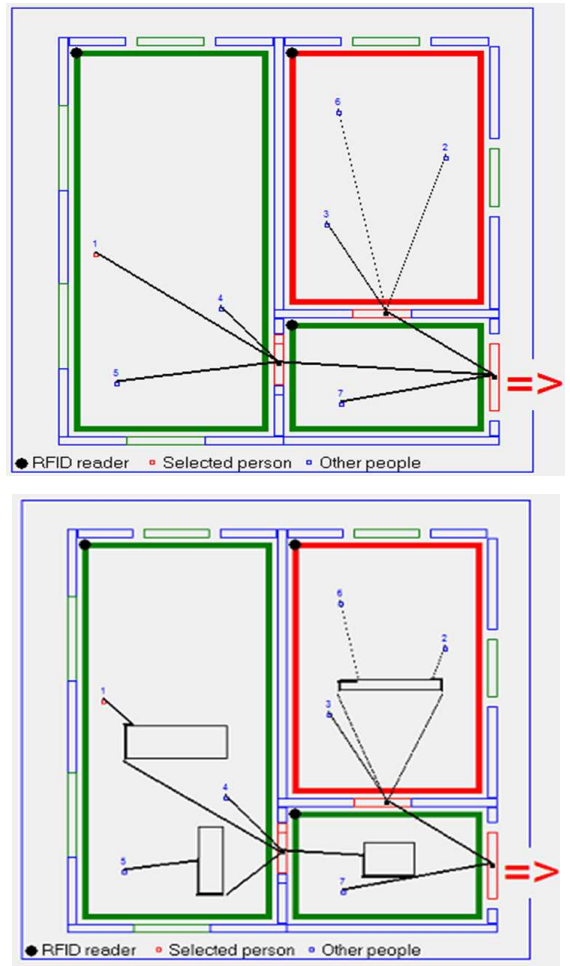

Fig. 6. Building navigation path and evacuation decision model.

\section{Conclusion}

The work presented in this paper is a contribution to the enhancement of the integration of RFID and WSN technologies using hybrid intelligent decision support systems to extend identification, tracking and sensing capabilities required for intelligent monitoring of activities and interaction support to people in emergency response when evacuating after hazard occurrence. These two technologies extend the penetration of pervasive computing in every domain of context aware environment reducing the boundaries of informal data and processes to reflect real time situations and instantaneous decision making. 
The proposed conceptual framework demonstrates that integrated RFID-WSNs coupled with the use of homogeneous and heterogeneous smart devices integrating readers can increase the robustness, the accuracy and reliability of data in the intelligent monitoring of smart buildings.

\section{Acknowledgments}

We would like to thank the Saudi High Education Ministry and the Brunel University (UK) for the support of the research grant, and Jerjer Hydrid intelligent Decision Systems for their software development contribution.

\section{References}

[1] K. Finkenzeller, in: Animal Identification, RFID Handbook Second Edition, 2003, p. 364.

[2] Considerations for High-Performance Toll Systems, TransCore Marketing Communications, Dallas 2002.

[3] A. Mainwaring, J. Polastre, R. Szewczyk, D. Culler, J. Anderson, in: 1 ACM Int. Workshop on Wireless Sensor Networks and Applications, Atlanta 2002, ACM, New York 2002, p. 88.

[4] Zhongmin Wang, Zhen Song, Peng-Yu Chen, Anisha Arora, Kevin Moore, YangQuan Chen, MASmote - A Mobility Node for MASnet (Mobile Actuator Sensor Networks), Utah State University, Logan 2004.

[5] M. Weiser, Scientific American September, 94 (1991).

[6] Jaekyu Cho, Yoonbo Shim, Taekyoung Kwon, Yanghee Choi, Sngheon Pack, Sooyeon Kim, IEEE Trans. Wireless Commun. 14, 50 (2007).

[7] R. Want, Computer 37, 84 (2004); IEEE Comput. Soc. 41, 21 (2008)
[8] Doohyun Ko, Bumjin Kim, Sunshin An, KSII Trans. Internet Inf. Syst. 4, 776 (2010).

[9] H. Liu, M. Bolic, A. Nayak, I. Stojmenovic, IEEE Network, 26 (2008).

[10] H. Yang, S.H. Yang, Measur. Control 40, 56 (2007).

[11] L. Zhang, Z. Wang, in: Proc. 5th Int. Conf. Grid Coop. Computer. Workshops (GCCW'06), 2006 , p. 463.

[12] T. Sanchez, D. Kim, in: Handbook on Mobile and Ubiquitous Computing: Innovations and Perspective, Eds.: L.T. Yang, E. Syukur, S.W. Loke, CRC, 2012.

[13] M. Ananthi, M.R. Sumalatha, in: Int. Advance Computing Conf. (IACC), IEEE 3rd International, 2013, p. 605.

[14] B. Torres, Q. Pang, G.W. Skelton, S. Bridges, N. Meghanathan, IJASUC 4, 1 (2010).

[15] A.V. Nedelcu, V.C. Stoianovici, I. Szekely, Bull. Transilv. Univ. Braşov Ser. I Eng. Sci. 4, 127 (2011).

[16] S. Padmapriya, I. Goel, A. Sunitha, P. Arul, IOSRJCE 16-2 V.3, 8 (2014).

[17] K. Opasjumruskit, T. Thanthipwan, O. Sathusen, P. Sirinamarattana, P. Gadmanee, E. Pootarapan, N. Wongkomet, A. Thanachayanont, M. Thamsirianunt, IEEE Pervasive Comput. Mag. 5, 54 (2006).

[18] F. Alshahrany, Zedan, I. Moualek, A Conceptual Framework for Small WSN Configuration using Intelligent Decision Support Systems, INTECH, London 2013.

[19] F. Alshahrany, Zedan, I. Moualek, J. Data Process. 4, 10 (2014)

[20] I. Moualek, Ph.D. Thesis, University of Nottingham, 1997. 